\title{
Puesta en Práctica de ODS en Educación Superior a través de la competencia "Sensibilidad hacia temas Medioambientales y Sociales" Implementation of SDGs in Higher Education through the competence "Sensitivity towards Environmental and Social issues"
}

\author{
María Luz Martín Peña, Eloísa Díaz Garrido y Cristina García Magro \\ luz.martin@urjc.es, eloisa.diaz@urjc.es,cristina.garcia.magro@urjc.es \\ Departamento Economía de la Empresa \\ Universidad Rey Juan Carlos \\ Madrid, España
}

\begin{abstract}
Resumen- El mundo en el que vivimos tiene que ser sostenible. Grandes cambios derivados de la producción y el consumo en masa, han llevado a deterioros medioambientales evidentes en el planeta. El desarrollo sostenible es una necesidad que es preciso abordar desde todos los ámbitos, económico, político, social, educativo. Las universidades, como centros de formación y de $\mathrm{I}+\mathrm{D}+\mathrm{i}$, pueden ser una pieza clave en acciones dirigidas al desarrollo sostenible. Así, la integración de los objetivos de desarrollo sostenible (ODS) en los centros de educación superior, pasa por introducir competencias alineadas con estos objetivos. En el caso de la asignatura Dirección de Producción, aparece una relación directa entre los contenidos que se abordan en la asignatura y el ODS 12 centrado en "garantizar modalidades de consumo y producción sostenible". Se realiza una propuesta que integra este objetivo en los contenidos de la asignatura y que permite alcanzar la competencia general "sensibilidad hacia temas medioambientales y sociales". La puesta en práctica se realiza con la metodología de aula invertida. De esta forma, se consigue facilitar al estudiante una visión integradora de los principios y ODS en su curriculum, aplicando una innovación educativa mediante la metodología activa que representa el aula invertida.
\end{abstract}

Palabras clave: Competencias, Educación para el Desarrollo Sostenible, Aula Invertida, ODS, Dirección de Operaciones

\begin{abstract}
The world we live in has to be sustainable. Great changes derived from mass production and consumption have led to evident environmental deterioration on the planet. Sustainable development is a necessity which must be addressed from all areas, economic, political, social, educational. Universities, as training and $\mathrm{I}+\mathrm{D}+\mathrm{i}$ centers, can be a key player in actions aimed at sustainable development. Thus, the integration of the Sustainable Development Goals (SDGs) in higher education centers involves introducing competencies aligned with these goals. In the case of the Production Management subject, there is a direct relationship between the contents that are addressed in the subject and the 12 SDG focused on "guaranteeing sustainable consumption and production modalities". A proposal is made that integrates this objective in the contents of the subject and that allows reaching the general competence "sensitivity towards environmental and social issues". The implementation is carried out with the flipped classroom methodology. In this way, it is
\end{abstract}

possible to provide the student with an integrative vision of the principles and SDGs in their curriculum, applying an educational innovation through the active methodology that the flipped classroom represents.

Keywords: Competencies, Education for Sustainable Development, Flipped Classroom, SDGs, Operations Management

\section{INTRODUCCIÓN}

Los planes de estudio de las titulaciones en el Espacio Europeo de Educación Superior (EEES) pretenden acercar a los alumnos a la realidad empresarial, involucrarles más activamente en su proceso de aprendizaje y proporcionar un marco favorable para la adquisición de diversas competencias. Éstas deben ser adquiridas por los estudiantes a lo largo del período formativo, trabajando en cada asignatura un conjunto de ellas.

Las asignaturas relativas a la materia Dirección de Producción y Operaciones incorporan competencias generales y específicas relacionadas directamente con el área de funcional de producción. En algunas guías docentes de estas asignaturas en distintas Universidades, se incluye una competencia relacionada con la "sensibilización hacia los temas medioambientales". Esta competencia representa de manera directa un objetivo importante de desarrollo sostenible incluido en la Agenda 2030 de la Unesco, es el ODS 12 relativo a garantizar modalidades de consumo y producción sostenible.

Desde la CRUE (Confederación de Rectores de las Universidades Españolas) se considera que la Universidad es un elemento «fundamental y necesario» para alcanzar los Objetivos de Desarrollo Sostenible (ODS). En este sentido realizan una propuesta de acciones de sensibilización para la implementación de la Agenda $2030^{1}$, entre las que se encuentra la Sostenibilización curricular, a través del desarrollo de Métodos docentes participativos y el desarrollo de contenidos específicos en las asignaturas.

\footnotetext{
${ }^{1} \mathrm{https}: / /$ www.crue.org/wp-

content/uploads/2021/01/Informe_Universidades_Crue-

Agenda2030.pdf
} 
"Es en este contexto donde las universidades tienen el reto de incluir los 17 Objetivos de Desarrollo Sostenible (ODS) en la amplia gama de sus ofertas formativas y se espera que la educación superior aporte conocimiento e innovación para hacer frente a los desafíos sociales, económicos y ambientales a través de la formación tanto del personal académico como de los estudiantes" (Chaleta, et al. 2021, pp.2).

Annan-Diab y Molinari (2017), en el contexto de un MBA, mencionan la importancia de incorporar el desarrollo sostenible en todo el curriculum desde una perspectiva integrada y multidisciplinar, reconociendo el valor añadido de perspectivas diferentes en sostenibilidad. Consideramos igualmente importante introducir el concepto de desarrollo sostenible también en estudios de Grado.

En este trabajo se presenta una experiencia de innovación educativa, basada en el aula invertida, que permita a los estudiantes adquirir la competencia "sensibilización hacia temas medioambientales y sociales" y a la vez la puesta en práctica de los ODS en una asignatura de grado.

Para ello, se eligió la asignatura de Dirección de Producción ya que tiene una relación directa con los temas medioambientales, y por tanto con los principios y objetivos de desarrollo sostenible En concreto, muchas de las metas incluidas en el ODS 12 se ven reflejadas en esta asignatura, tales como: "gestión sostenible y el uso eficiente de los recursos naturales", "reducir la generación de desechos mediante actividades de prevención, reducción, reciclado y reutilización", "alentar a las empresas, en especial las grandes empresas y las empresas transnacionales, a que adopten prácticas sostenibles e incorporen información sobre la sostenibilidad en su ciclo de presentación de informes".

De esta forma, se realiza un planteamiento actual hacia la materia dirección de operaciones sostenible. Las operaciones sostenibles integran la orientación tradicional de Dirección de operaciones hacia la eficiencia $y$ el beneficio, con consideraciones más amplias de los stakeholders internos y externos y su impacto medioambiental (Kleindorfer et al., 2005).

\section{Contexto}

La asignatura Dirección de Producción del Grado en Administración y Dirección de Empresas, aborda el conocimiento del área de producción y operaciones en la empresa manufacturera, la función de producción con enfoque estratégico y toma de decisiones vinculadas a producto, proceso, capacidad, localización y calidad, con un marcado carácter teórico-práctico. Pero falta un planteamiento en el que la producción y las operaciones sostenibles, se aborden con un enfoque integrador.

La competencia general sensibilización hacia los temas medioambientales y sociales surge como clave en un entorno como el actual, dado que la protección del medio ambiente se convierte en una prioridad para los actores económicos y sociales. Así, la sociedad actual está preocupada por la degradación del medio ambiente como consecuencia de la actividad industrial ya que supone el consumo de una gran cantidad de recursos y de energía, y da lugar a riesgos medioambientales globales que requieren soluciones inmediatas (Rezaee y Elam, 2000; Banerjee, 2001). Esta creciente preocupación social provoca cambios en el entorno que rodea a la empresa, exigiéndole acciones significativas en sus prácticas productivas y de gestión (Bansal y Roth, 2000). La sostenibilidad medioambiental se convierte en objetivo para muchas de ellas (Huppes e Isikawa, 2007; Prajogo, et al., 2012). $\mathrm{Y}$ es un objetivo de desarrollo sostenible.

Desde diferentes ámbitos la empresa recibe presiones para apostar por la sostenibilidad medioambiental:1) Desde el mercado, tanto por el entorno genérico como por el específico del sector al que pertenece la empresa; 2) Desde la sociedad, a través de la opinión pública en general y asociaciones y organizaciones no gubernamentales; 3) desde el sector financiero, a través de inversores, proveedores de fondos y compañías de seguros, 4) Desde la administración pública (Fineman y Clarke, 1996).

Cabe preguntarse, ¿cómo trabajar estos contenidos, competencias y el enfoque de principios y ODS en la asignatura? Estudios previos han demostrado que la aplicación de metodologías tradicionales basadas en clases magistrales, no consiguen que el alumno adquiera ciertas competencias (Martín-Peña, et al. 2011), por lo que se hace necesario desarrollar metodologías más novedosas (Mingorance y Calvo, 2013) para encaminar a los estudiantes hacia la familiarización con los temas medioambientales.

\section{A. Objetivos}

Los objetivos que se pretenden alcanzar con esta iniciativa de innovación educativa son:

Conseguir que los alumnos tengan una visión global e integradora de la función de producción $\mathrm{y}$ operaciones sostenible, así como de los principios y ODS.

Incentivar la participación e implicación de los alumnos mediante el planteamiento de problemas reales y motivadores.

\section{B. Público Objetivo}

La experiencia de innovación educativa que se presenta ha sido desarrollada durante el curso 2018-2019, en la asignatura "Dirección de Producción" que se imparte en el segundo curso del Grado en Administración y Dirección de Empresas. Se trata de una asignatura obligatoria, de 6 créditos y cuyo tiempo de trabajo dentro del aula queda distribuido en 30 horas de clases teóricas y 25 horas de clases prácticas. Han participado tres grupos de docencia con un total de 140 alumnos y 3 profesores.

\section{DESCRIPCIÓN}

\section{A. Metodología. Flipped Classroom}

En los últimos años, cada vez es más habitual llevar a la práctica estrategias didácticas innovadoras que permitan la participación activa del estudiante y les encamine hacia los beneficios de ser responsables de su propio proceso de aprendizaje.

En este sentido, Flipped Classroom o "aula invertida" se ha convertido en una metodología ampliamente estudiada y aplicada en el contexto de educación superior (Akçayır y Akçayır, 2018).

Sohrabi y Iraj (2016, pp.514) definen Flipped Classroom como "un modelo de instrucción donde lo que se ha hecho tradicionalmente en clase se traslada a actividades en casa y lo 
que se ha hecho tradicionalmente en casa, se transfiere como actividades de clase".

Se trata de un enfoque que combina el aprendizaje autónomo basado en videos, lecturas etc fuera del aula, para utilizar el tiempo de clase en actividades de aprendizaje activo, social, de discusión y resolución de problemas (Abeysekera y Dawson, 2015; Rotellar y Cain, 2016; Lo y Hew, 2017; Akçayır y Akçayır, 2018)

El sello distintivo del Flipped Classroom, por tanto, es el compromiso con el contenido a través de la práctica, proyectos, resolución de problemas y colaboración con compañeros (Blair et al., 2016).

Ahora bien, tal y como establece Bristol (2014), antes de poner en práctica esta metodología es necesario delimitar las competencias que se pretenden conseguir del proceso de enseñanza-aprendizaje centrado en el alumno, que combine los contenidos teóricos con la práctica fundamentada en problemas de la vida real. Por ello, se hace necesario definir los objetivos de aprendizaje que se pretenden alcanzar, para posteriormente, organizar las actividades y tareas antes, durante y después de la clase (Gilboy et al., 2014).

Se ha demostrado que esta metodología didáctica impacta positivamente en la confianza, motivación y compromiso de los estudiantes (Awidi y Paynter, 2019) ya que el tiempo de clase se convierte en una experiencia de aprendizaje similar a la práctica real. De este modo, el nivel de motivación influye en el FOCUS y en el nivel de esfuerzo que los alumnos dedican a las actividades de aprendizaje (Cole et al., 2004).

En el ámbito educativo, generalmente la tendencia ha sido motivar al alumnado de manera extrínseca, descuidando que, si se incentiva la motivación intrínseca, las recompensas adquiridas pueden ser mayores. Así actúa el aula invertida. Gross et al., (2015) muestran que el entorno de flipped classroom incentiva el compromiso con el material ofrecido, implica al alumno en su proceso de aprendizaje a través del esfuerzo, ofrece una preparación más precisa $y$, en definitiva, mejora los resultados académicos

\section{B. Actividades y Técnicas}

La experiencia se enmarca en las prácticas obligatorias de las asignaturas. Se forman grupos de trabajo entre los alumnos.

Los temas que se abordan son la producción sostenible, la logística inversa y el vehículo eléctrico. En cada uno de los temas se les facilita información básica de partida, y se les activa mediante preguntas, cuya respuesta exige diferentes acciones. Así, en grupos formados por 4 personas, tienen que buscar documentación, realizar propuestas, discutirlas, exponerlas y llegar a conclusiones. Todo ello con retroalimentación entre los grupos y el profesor, que ejerce de moderador.

Para el tema producción sostenible, el objetivo es que los estudiantes conozcan el concepto y su importancia en la consecución de algunos objetivos de desarrollo sostenible. Los grupos reciben información con ideas básicas de lo que es la producción Sostenible, con ejemplos de empresas que apuestan por la misma (Inditex, Meliá, Vega Sicilia, Tesla...). Trabajan fuera del aula esta información y tienen que buscar más información para responder una serie de cuestiones, así, se les pide que identifiquen aspectos en común y diferencias de la Producción Sostenible en cada empresa y que respondan a ¿qué implicaciones estratégicas y operativas suponen para la empresa la Producción Sostenible? En grupo, en el aula, se discuten las respuestas y obtienen conclusiones. Como trabajo autónomo cada alumno entrega ensayo crítico de la lectura "Consumo y Producción Sostenible".

Con el topic logística inversa se busca presentar la misma como un camino hacia la Producción Sostenible. El objetivo es conocer qué es logística inversa y su importancia en la actualidad para la consecución del desarrollo sostenible, y como nuevo modelo de negocio. Sobre el caso real del reciclaje de aparatos eléctricos, los alumnos tienen que buscar información para el caso concreto de móviles, en base a las siguientes ideas: ¿Qué se hace para el reciclaje de dispositivos móviles? Describir distintas iniciativas. Propuestas para conseguir una producción sostenible y reciclado para el caso de los dispositivos móviles. En grupo, en el aula, se discuten las respuestas y obtienen conclusiones. Como trabajo autónomo cada alumno entrega ensayo crítico de la lectura "Gestión de residuos en aparatos eléctricos y electrónicos".

El tema vehículo eléctrico se aborda desde la perspectiva de la producción y el consumo. El objetivo es conocer como la respuesta a la normativa medioambiental, lleva al sector de automoción al nuevo producto que representa el coche eléctrico, con muchas implicaciones en el modelo de negocio. También las motivaciones e implicaciones hacia la compra y uso de un vehículo eléctrico. Desde un video que ilustra la producción del coche eléctrico, los alumnos deben buscar información sobre el impacto de la misma en el medio ambiente, así como sobre los nuevos modelos de negocio y consumo en torno al coche eléctrico, incluyendo las plataformas digitales de carsharing. En grupo, en el aula, se discuten las respuestas y obtienen conclusiones.

Al finalizar la asignatura, se procedió a recoger información de los alumnos mediante un cuestionario anónimo. Éste quedó formado por 5 preguntas (a su vez compuestas de varios ítems), que permiten valorar distintos aspectos como: la importancia de la asignatura en la sostenibilidad, las actividades desarrolladas, la adquisición de competencias, la metodología desarrollada y los resultados de aprendizaje alcanzados. Se utilizó para la valoración de los ítems una escala Likert de 1- poco importante a 10 -muy importante). Además, se incluía una pregunta de valoración global de la experiencia.

\section{Resultados}

Se obtuvieron un total de 140 cuestionarios válidos.

En primer lugar, hay que destacar que todos los alumnos participantes consideran que el área de producción y operaciones tiene una gran importancia en la protección del medio ambiente $(8,91)$, la consecución de ventajas económicas $(9,58)$ y en la consecución del ODS número $12(9,41)$. Siendo la asignatura de Dirección de Producción la única en la que han tratado y trabajado la competencia "Sensibilización hacia temas medioambientales" en las titulaciones de ADE.

Los aspectos mejor valorados (por encima de 9,5) han resultado ser los contenidos en general y las actividades asociadas al vehículo eléctrico en particular. Por el contrario, las actividades menos valoradas son las que el alumno debe realizar fuera de clase (valor medio 7,9), debido a que suponen un mayor esfuerzo y trabajo autónomo por parte del estudiante. 
Las competencias que los alumnos consideran que han adquirido de forma más sólida (puntuación en media superior a 8 sobre 10) son las relativas a la capacidad de trabajo en grupo y la capacidad de razonamiento crítico, además de la competencia sensibilidad hacia temas sociales y medioambientales. En cambio, la competencia menos desarrollada (valor medio inferior a 7 sobre 10) es la relativa a la capacidad de resolución de problemas.

En lo relativo a la metodología de clase inversa, los resultados ponen de manifiesto que resulta ser una metodología muy bien valorada y que mejora el uso de todos los recursos didácticos utilizados. Además, ha resultado ser una metodología muy eficaz. Los profesores son muy bien valorados (con medias superiores a 9) en cuanto a su aportación y dedicación. Las actividades que se proponen para que los alumnos las realicen fuera de clase sigue siendo el aspecto peor valorado (media cercana a 7,5), además la eficacia o utilidad de las actividades fuera de clase generan mayor controversia o desacuerdo al presentar mayor variabilidad (desviación típica superior a 2).

En cuanto a los resultados de aprendizaje conseguidos, el alumno valora que la experiencia está diseñada para hacerle pensar y descubrir cuestiones por mí mismo antes de que sean explicadas por el profesor $(8,66)$.

En términos generales la valoración global de la experiencia desarrollada es excelente, ya que más del $80 \%$ de los alumnos lo aprecian de forma "sobresaliente" (puntuación 9 o 10).

La tabla 1 recoge el resumen de los resultados obtenidos a través de los datos recogidos mediante el cuestionario, mostrando los valores medios, mediana, moda y desviación típica para cada uno de los ítems del cuestionario.

Tabla 1: Resultados valoración estudiantes experiencia

\begin{tabular}{lllll}
\hline & Media & Mediana & Moda & DTípi \\
\hline Importancia DPO & & & & \\
- Protección medio amb & 8.91 & 9 & 9 & 0,75 \\
- Ventajas económicas & 9,58 & 9 & 9 & 0,65 \\
- Consecución ODS 12 & 9,41 & 9,5 & 9 & 0,79 \\
\hline Valoración & & & & \\
- Contenidos & 9,66 & 10 & 10 & 0,63 \\
- Actividades aula & 9,33 & 9 & 9 & 0,63 \\
- Actividades fuera aula & 7,91 & 9 & 9 & 2,1 \\
\hline Competencias & & & & \\
- Razonamiento crítico & 8,33 & 8,5 & 8 & 1,34 \\
- Trabajo equipo & 8 & 8 & 8 & 1,38 \\
- Análisis información & 7,33 & 7,5 & 8 & 1,40 \\
- Organ./ Planificación & 7,58 & 8 & 8 & 1,58 \\
-Sensibilidad med.amb & 8,25 & 8,5 & 8 & 1,67 \\
- Resolución problemas & 6,75 & 6,5 & 5 & 1,56 \\
\hline $\begin{array}{l}\text { Metodología } \\
\text {-Desarrollo nuevas }\end{array}$ & 9,5 & 10 & 10 & 0,78 \\
capacidades & & & & \\
-Estímulo aprendizaje & 9,4 & 10 & 10 & 0,77 \\
-Autonomía & & & & \\
aprendizaje & 9,5 & 10 & 10 & 0,77 \\
-Motivadora & 8,75 & 9 & 9 & 1,25 \\
\hline Rdos aprendizaje & & & & \\
- Mejora compromiso & 9,16 & 9,5 & 10 & 1,16 \\
medio ambiente & & & & \\
- He conocido temas & 8,66 & 9 & 9 & 0,66 \\
por mi mismo & & & & \\
\hline Valoración global & 9,66 & 10 & 10 & 0,63 \\
\hline
\end{tabular}

\section{CONCLUSIONES}

Con este trabajo se propone la puesta en práctica del ODS 12 relativo a "garantizar modalidades de consumo y producción sostenible", en la asignatura Dirección de Producción del Grado en Administración y Dirección de Empresas, a través de la adquisición de la competencia general "Sensibilización hacia temas medioambientales y sociales", incluida en el Libro Blanco de la titulación, y en muchas guías docentes de distintas universidades españolas.

La puesta en práctica de la competencia general citada, se ha realizado a través de la metodología de Flipped Classroom o Aula Invertida, lo que ha permitido trabajar además de la Sensibilización hacia temas medioambientales, otras competencias curriculares, como el trabajo en equipo, aprendizaje autónomo y capacidad de razonamiento crítico.

A través de temas actuales incluidos en la asignatura, como reciclaje, producción sostenible y vehículo eléctrico, se pone el foco en la sostenibilidad ambiental, teniendo los alumnos que buscar información, analizarla, sintetizarla, sacando conclusiones que se comparten en los grupos de trabajo.

Consideramos que la experiencia que se presenta tiene importantes implicaciones prácticas. En primer lugar, al referirse el eje central a un tema con una problemática medioambiental y social (desarrollo sostenible), se considera clave el papel protagonista del alumno en cuanto a la concienciación y propuestas de solución de la problemática mencionada. En segundo lugar, con esta experiencia se contribuye a la puesta en práctica de acciones de sensibilización dentro de las aulas universitarias para la implementación de la Agenda 2030 en relación con el cumplimento de los ODS.

Se aborda así en educación superior un ODS, el 12, distinto a los considerados clásicos en este ámbito, a saber, el 4, 5, 8 y 10.

En los próximos cursos, se pretende implantar de nuevo esta experiencia, con el fin de contar con datos que permitan realizar estudios comparativos respecto de la valoración de la misma por parte de los estudiantes, como de la adquisición de esta competencia y otras relacionadas, a través de resultados de aprendizaje centrados en ella.

\section{REFERENCIAS}

Abeysekera, L. y Dawson, P. (2015). Motivation and cognitive load in the flipped classroom: definition, rationale and a call for research. Higher education research \& development, 34(1), 1-14.

Akçayır, G. y Akçayır, M. (2018). The flipped classroom: A review of its advantages and challenges. Computers \& Education, 126, 334-345.

Annan-Diab, F. y Molinari, C. (2017). Interdisciplinarity: Practical approach to advancing education for sustainability and for the Sustainable Development Goals. International Journal Management Education, 15, 73-83.

Awidi, I. T. y Paynter, M. (2019). The impact of a flipped classroom approach on student learning experience. Computers \& Education, 128, 269-283.

Banerjee, S.B. (2001). Managerial perceptions of corporate environmentalism: Interpretations from industry and 
strategic implications for organizations. Journal of Management Studies 38, 489-513.

Bansal, P. y Roth, K. (2000). Why companies go green: a model of ecological responsiveness. Academy of Management Journal, 43, 717-736.

Blair, E., Maharaj, C. y Primus, S. (2016). Performance and perception in the flipped classroom. Education and information Technologies, 21(6), 1465-1482.

Bristol, T. J. (2014). Flipping the Classroom. Teaching and Learning in Nursing, 9(1), 43-46.

Cole, M., Feild, H. y Harris, S. (2004). Student learning motivation and psychological hardiness: Interactive effects on students' reactions to a management class. Academy of Management Learning and Education, 3(1), 64-85.

Chaleta, E., Saraiva, M., Leal, F., Fialho, I. y Borralho, A. (2021). Higher Education and Sustainable Development Goals (SDG)-Potential Contribution of the Undergraduate Courses of the School of Social Sciences of the University of Évora. Sustainability, 13 (4), 1828.

Gilboy, M. B., Heinerichs, S. y Pazzaglia, G. (2015). Enhancing student engagement using the flipped classroom. Journal of nutrition education and behavior, 47(1), 109-114.

Gross, B., Marinari, M., Hoffman, M., DeSimone, K. y Burke, P. (2015). Flipped@ SBU: Student satisfaction and the college classroom. Educational Research Quarterly, $39(2), 36$.

Huppes, G. y Ishikawa, M. (2007). Sustainability evaluation: diverging routes recombined? Tasks for a new Working Group on Modelling and Evaluation for Sustainability. Journal of Cleaner Production, 15 (11-12), 1183-1184.

Kleindorfer, P.R., Singhal, K. y Van Wassenhove, L.N. (2005). "Sustainable operations management." Production and Operations Management, 14(4), 482-492.
Lo, C. K., y Hew, K. F. (2017). A critical review of flipped classroom challenges in K-12 education: Possible solutions and recommendations for future research. Research and practice in technology enhanced learning, 12(1), 1-22.

Martín-Peña, M.L., Díaz-Garrido, E., Castillo, B., Barrio del, L. (2011). Estudio comparativo de cambios metodológicos y percepción del alumno en la materia de Dirección de producción y operaciones para la adquisición de competencias en el proceso de adaptación al EEES. Revista de Formación e Innovación Educativa Universitaria, 4(2), 126-144.

Mingorance, C. y Calvo, A. (2013). Los resultados de los estudiantes en un proceso de evaluación con metodologías distintas. Revista de Investigación Educativa, 31 (1), 275 293.

Prajogo, D., Tang, A. K. y Lai, K. H. (2012). Do firms get what they want from ISO 14001 adoption?: an Australian perspective. Journal of Cleaner Production, 33, 117-126.

Rezaee, Z. y Elam, R. (2000). Emerging ISO 14000 environmental standards: a step-by-step implementation guide. Managerial Auditing Journal, 15 (1/2), 60-67.

Rotellar, C. y Cain, J. (2016). Research, perspectives, and recommendations on implementing the flipped classroom. American journal of pharmaceutical education, 80(2), 19.

Sohrabi, B. y Iraj, H. (2016). Implementing flipped classroom using digital media: A comparison of two demographically different groups perceptions. Computers in Human Behavior, 60, 514-524. 